\title{
Multipliers and Cyclic Vectors in the Bloch Space
}

\author{
LEON BROWN \& A. L. SHIELDS*
}

\section{Introduction}

In this paper we study the cyclic vectors in $B$, the Bloch space with the weak* topology, and in $\Theta_{0}$, the "little" Bloch space with the norm topology. A result is obtained which implies that every outer function in $B\left(\Theta_{0}\right)$ is cyclic. We also obtain a simple characterization of multipliers in $B$ and $B_{0}$.

The Bloch space $B$ in the open unit disc $D$ in the complex plane is the space of all those analytic functions $f$ such that $\left(1-|z|^{2}\right) f^{\prime}(z)$ is bounded in $D$. We norm $B$ as follows:

$$
\|f\|=|f(0)|+\sup \left\{\left(1-|z|^{2}\right)\left|f^{\prime}(z)\right|: z \in D\right\} .
$$

With this norm $\mathscr{B}$ is a Banach space and $\Theta_{0}$ a closed subspace. Here $B_{0}$, sometimes called the "little" Bloch space, denotes the set of those $f$ in $B$ for which $\left(1-|z|^{2}\right) f^{\prime}(z) \rightarrow 0$ as $|z| \uparrow 1$. For information about $B$ and $\Theta_{0}$, see [1] and [2].

The space $B$ with the norm (1) is isometric to the second dual $\Theta_{0}^{* *}$ (see [9]). Furthermore, the polynomials are norm dense in $\beta_{0}$ and in $\Theta_{0}^{*}$, and are weak* dense in $B$. Note that $B$ is not norm separable.

We have a growth estimate for Bloch functions (see, e.g., [3, Eq. (4)]):

$$
|f(z)| \leq\left\{1+\log \frac{1}{1-|z|}\right\}\|f\| .
$$

Thus $B$ is contained in $L_{a}^{p}$ (the analytic $L^{p}$ functions in $D$ ) for $p<\infty$. For the Hardy spaces we have $H^{\infty} \subset \Re$, but $H^{p}$ is not contained in $B$ for any $p<\infty$; also, $\mathbb{B}$ is not contained in the Nevanlinna class.

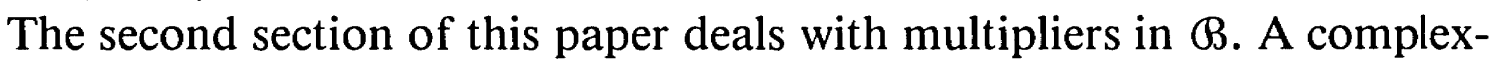
valued function $\phi$ in $D$ is called a multiplier on $B$ if $\phi \cap \subset \cap$. By $M_{\phi}$ we denote the operator of multiplication by $\phi: M_{\phi} f=\phi f(f \in ß)$. The set of all multipliers will be denoted by $M(\beta)$. An application of the closed graph theorem shows that $M_{\phi}$ is a bounded linear transformation on $B$. Hence it has a finite norm $\left\|M_{\phi}\right\|$.

Received February 21, 1990. Revision received September 24, 1990.

* Deceased. The research of this author was supported in part by the National Science Foundation.

Michigan Math. J. 38 (1991). 
In the third section we study (weak*) cyclic vectors for the space $\beta$. These are the function $f$ in $\mathbb{B}$ whose polynomial multiples are weak* dense in $B$ (i.e., they are cyclic vectors in the weak* topology for the operator of multiplication by $z$ on $($ ). Note that a duality argument yields the fact that if $f$ is in $\Re_{0}$, then $f$ is (norm) cyclic in $\Theta_{0}$ if and only if it is weak* cyclic in $ß$. When we refer to cyclic vectors in $B$, the weak* topology is always understood.

In [3], Anderson, Fernández, and Shields show that if $f$ is outer and bounded, then $f$ is cyclic. Theorem 3 shows that the outer requirement alone is enough to ensure that a Bloch space function is cyclic.

\section{Multipliers in the Bloch Spaces}

THEOREM 1. The following are equivalent:

(a) $\phi$ is a multiplier on $B$;

(b) $\phi$ is a multiplier on $\AA_{0}$;

(c) $\varphi \in H^{\infty}$ and

$$
\left|\phi^{\prime}(z)\right|=O\left(\frac{1}{(1-|z|) \log (1 /(1-|z|))}\right) .
$$

Proof. (a) $\Rightarrow$ (c) We thank the referee for suggesting the following proof; our original proof was more complicated.

Suppose that $\varphi$ is a multiplier of $B$. Then by [5, Lemma 11] $\varphi \in H^{\infty}$ and $|\varphi(z)| \leq\left\|M_{\varphi}\right\|$. Let $z_{0} \in D \backslash\{0\}$ be arbitrary. We complete the proof of the implication $(\mathrm{a}) \Rightarrow(\mathrm{c})$ by showing

$$
\left|\varphi^{\prime}\left(z_{0}\right)\right| \leq \frac{4\left\|M_{\varphi}\right\|}{\left(1-\left|z_{0}\right|\right) \log \left(1 /\left(1-\left|z_{0}\right|\right)\right)} .
$$

Let $z_{0}=r e^{i \theta}$. Since $L(z)=\log \left(1-e^{-i \theta} z\right)$ is in $\Theta$ and $\varphi$ is a multiplier of $\Theta$, we have

It follows that

$$
\|\varphi L\| \leq\left\|M_{\varphi}\right\|\|L\|=2\left\|M_{\varphi}\right\| .
$$

Hence

$$
\begin{aligned}
\left(1-|z|^{2}\right)\left|\varphi^{\prime}(z) L(z)\right| & \leq 2\left\|M_{\varphi}\right\|+\left(1-|z|^{2}\right)\left|\varphi(z) L^{\prime}(z)\right| \\
& \leq 2\left\|M_{\varphi}\right\|+2\|\varphi\|_{\infty} \leq 4\left\|M_{\varphi}\right\| .
\end{aligned}
$$

$$
\left|\varphi^{\prime}(z)\right| \leq \frac{4\left\|M_{\varphi}\right\|}{\left(1-|z|^{2}\right)\left|\log \left(1-e^{-i \theta} z\right)\right|} .
$$

To see that $(*)$ holds set $z=z_{0}$ and replace $1-\left|z_{0}\right|^{2}$ with the smaller value $1-\left|z_{0}\right|$.

(b) $\Rightarrow$ (c) Given $z_{0}=r e^{i \theta} \neq 0$ and $\alpha$ in $(0,1)$, let

$$
L_{\alpha}(z)=\left(\frac{1}{z} \log \left(1-e^{-i \theta} z\right)\right)^{\alpha} .
$$


A calculation shows that $L_{\alpha} \in \Re_{0}$ and $\sup _{\alpha}\left\|L_{\alpha}\right\|=k<+\infty$. In a manner similar to the proof of $(\mathrm{a}) \Rightarrow(\mathrm{c})$, one obtains that if $\varphi$ is a multiplier of $\beta_{0}$ then, for each $\alpha$,

Hence,

$$
\left|\varphi^{\prime}\left(z_{0}\right)\right| \leq \frac{2 k\left\|M_{\varphi}\right\|}{\left(1-\left|z_{0}\right|^{2}\right)\left|\left(\log \left(1-\left|z_{0}\right|\right) / z_{0}\right)^{\alpha}\right|} .
$$

$$
\left|\varphi^{\prime}\left(z_{0}\right)\right| \leq \frac{2 k\left\|M_{\varphi}\right\|}{\left(1-\left|z_{0}\right|\right) \log \left(1 /\left(1-\left|z_{0}\right|\right)\right)} .
$$

(c) $\Rightarrow$ (a) Assume $f \in \mathcal{B}, \varphi \in H^{\infty}$, and

$$
\left|\varphi^{\prime}(z)\right|=O\left(\frac{1}{(1-|z|) \log (1 /(1-|z|))}\right) .
$$

Note that $(\varphi f)^{\prime}=\varphi f^{\prime}+\varphi^{\prime} f$ and we have

$$
\left|\left(\varphi f^{\prime}\right)(z)\right| \leq\|\varphi\|_{\infty}\left|f^{\prime}(z)\right| \leq C \frac{1}{1-|z|} .
$$

For $|z| \geq \frac{1}{2}$ we have $|f(z)| \leq C \log (1 /(1-|z|))$, which implies $\left|\left(\varphi^{\prime} f\right)(z)\right| \leq$ $C /(1-|z|)$. Thus $\varphi f \in \mathbb{B}$.

(c) $\Rightarrow$ (b) One can easily show that if $f \in \Re_{0}$ then $f(z)=o(\log (1 /(1-r)))$. Using this fact, the proof is similar to the proof of $(c) \Rightarrow(a)$.

LEMMA 1 (see [3, Lemma 1] or [4, Prop. 2]).

(a) If $\left\{f_{n}\right\} \subset B$ then $f_{n} \rightarrow 0$ weak ${ }^{*}$ if and only if $f_{n}(z) \rightarrow 0$ for all $z$ in $D$, and $\sup \left\|f_{n}\right\|<\infty$.

(b) If $\left\{f_{\alpha}\right\} \subset \mathcal{B}, 0 \leq \alpha<1$, then $\lim f_{\alpha} \rightarrow 0$ (as $\alpha \uparrow 1$ ) weak ${ }^{*}$ if and only if $\lim f_{\alpha}(z) \rightarrow 0(\alpha \uparrow 1)$ for all $z$ in $D$, and $\lim \sup \left\|f_{\alpha}\right\|<\infty$.

REMARK. Both (a) and (b) remain valid if $\Theta$ is replaced by $\Re_{0}$ and weak* is replaced by weakly.

Let $[f]$ denote the weak* (norm) closure in $\mathbb{B}\left(\Theta_{0}\right)$ of polynomial multiples of $f$. Set $\|f\|_{0}=\sup _{z}(1-|z|)|f(z)|$ if it is finite.

LEMMA 2. If $g \in M\left(\AA_{0}\right)=M(囚)$ then

(a) $f \in \Re_{0}$ implies $g f \in[f]$ (norm topology);

(b) $f \in \Re$ implies $g f \in[f]$ (weak* topology).

Proof. (a) We show that if $g \in M\left(\Re_{0}\right)$ and $f \in \Re_{0}$ then $g_{t}(f) \in[f]$, where $g_{t}(z)=g(t z)$. One easily shows that if $P_{n}$ is the partial sum of the power series for $g_{t}$ then $P_{n} f \rightarrow g_{t} f$ (norm). Thus we have $g_{t} f$ is in the closure of polynomial multiples of $f$, which implies $g_{t} f \in[f]$. For $z \in D, g_{t}(z) f(z) \rightarrow$ $g(z) f(z)$. Furthermore,

$$
\begin{aligned}
\left\|g_{t} f\right\| & =\left\|g_{t} f^{\prime}+f\left(g_{t}\right)^{\prime}\right\|_{0}+|g(0) f(0)| \\
& \leq\|g\|_{\infty}\|f\|+\left\|f\left(g_{t}\right)^{\prime}\right\|_{0}+|f(0) g(0)| .
\end{aligned}
$$


If $|z|=r$ and $|z| \geq \frac{1}{2}$, then

$$
\begin{aligned}
\left|f(z)\left(g_{t}(z)\right)^{\prime}\left(1-r^{2}\right)\right| & \leq C \log \left(\frac{1}{1-r}\right) t\left|g^{\prime}(t z)\right|(1-r) \\
& \leq C \log \left(\frac{1}{1-r}\right) \max _{|y|=r}\left|g^{\prime}(t y)\right|(1-r) \\
& \leq C \log \left(\frac{1}{1-r}\right) \max _{|y|=r}\left|g^{\prime}(y)\right|(1-r) \\
& \leq C \quad \text { (by Theorem 1). }
\end{aligned}
$$

Thus, by Lemma $1, g_{t} f \rightarrow g f$ weakly and the proof is complete.

(b) The proof is essentially the same for $f \in \mathbb{B}$; we omit the details.

\section{Cyclic Vectors in the Bloch Spaces}

LEMMA 3. If $f, g \in \mathbb{B}$ then

$$
\left\|\left(f-f_{t}\right) g_{t}^{\prime}\right\|_{0} \leq\left\|f^{\prime}\right\|_{0}\left\|g^{\prime}\right\|_{0} .
$$

Here $g_{t}(z)=g(t z)$ for all $z \in D$.

Proof. Since $f(z)-f(t z)=\int_{t z}^{z} f^{\prime}$ (integrating along the radius), we have

$$
\begin{aligned}
|f(z)-f(t z)| & \leq\left\|f^{\prime}\right\|_{0} \int_{r t}^{r}(1-\tau)^{-1} d \tau \\
& =\left\|f^{\prime}\right\|_{0} \log \frac{1-r t}{1-r} .
\end{aligned}
$$

Also, if $x=(1-r) /(1-t r)$ then

$$
\log \left(\frac{1-r t}{1-r}\right)\left(\frac{1-r}{1-t r}\right)=x \log \frac{1}{x}
$$

is bounded by $(1 / e) \log e$ for $0 \leq t \leq 1$ and $0 \leq r<1$.

We proceed with the proof as follows: If $0 \leq t \leq 1$ and $0 \leq r<1$ then

$$
\begin{aligned}
\left|(f(z)-f(t z)) t g^{\prime}(t z)\right|(1-r) & \leq\left\|f^{\prime}\right\|_{0} \log \left(\frac{1-r t}{1-r}\right)\left|g^{\prime}(t z)\right|(1-t r) \cdot \frac{1-r}{1-t r} \\
& \leq\left\|f^{\prime}\right\|_{0}\left\|g^{\prime}\right\|_{0} \log \left(\frac{1-r t}{1-r}\right) \frac{1-r}{1-t r} \\
& <\left\|f^{\prime}\right\|_{0}\left\|_{g^{\prime}}\right\|_{0} .
\end{aligned}
$$

LEMMA 4. If $f \in H^{\infty} \subset \Re, g \in \Re$, and $f g \in \Re$, then $f g \in[g]$.

Proof. For $z \in D,\left(f_{t} g\right)(z) \rightarrow(f g)(z)$. We have $\left(f_{t} g\right)^{\prime}=f_{t} g^{\prime}+g\left(f_{t}\right)^{\prime}$, and

$$
\begin{aligned}
\left\|\left(f_{t} g^{\prime}\right)\right\|_{0} & \leq\left\|f_{t}\right\|_{\infty}\left\|g^{\prime}\right\|_{0} \leq\|f\|_{\infty}\left\|g^{\prime}\right\|_{0} ; \\
\left\|\left(f_{t}\right)^{\prime} g\right\|_{0} & \leq\left\|\left(g-g_{t}\right) f_{t}^{\prime}\right\|_{0}+\left\|g_{t}\left(f_{t}\right)^{\prime}\right\|_{0} \\
& <\left\|g^{\prime}\right\|_{0}\left\|f^{\prime}\right\|_{0}+\left\|g f^{\prime}\right\|_{0} ;
\end{aligned}
$$


Thus

$$
\begin{aligned}
\left\|g f^{\prime}\right\|_{0} & =\left\|(f g)^{\prime}-\left(f g^{\prime}\right)\right\|_{0} \\
& \leq\left\|(f g)^{\prime}\right\|_{0}+\|f\|_{\infty}\left\|g^{\prime}\right\|_{0} .
\end{aligned}
$$

$$
\left\|\left(f_{t} g\right)\right\| \leq|f(0) g(0)|+\left\|f^{\prime}\right\|_{0}\left\|g^{\prime}\right\|_{0}+\left\|(f g)^{\prime}\right\|_{0}+2\|f\|_{\infty}\left\|g^{\prime}\right\|_{0}
$$

and $f_{t} g \rightarrow f g$ weak*, which completes the proof.

REMARK. If $g$ and $f g$ are in $\Re_{0}$ then $f_{t} g \rightarrow f g$ weakly and we have $f g \in[g]$ (norm).

THEOREM 2. If $f, g \in \mathbb{B},|f(z)| \geq|g(z)|$ in $D$, and $g$ is cyclic, then $f$ is cyclic.

Proof. We have $g / f \in H^{\infty}$ and $(g / f) f=g \in[f]$, which implies that $f$ is cyclic.

PROPOSITION 1 (see [3, Cor. to Prop. 1]).

(a) If $f$ is cyclic in $H^{\infty}$ (with the weak* topology) then $f$ is cyclic in 3. Note that $f$ is cyclic in $H^{\infty}$ if and only iff is outer (see [8, Thm. 5.5]).

(b) If $f$ is cyclic in $B$ then $f$ is cyclic in $L_{a}^{2}$.

REMARKS. (a) This result also follows from the fact that the identity $i$ : $H^{\infty} \rightarrow ß$ is weak*-weak* continuous and $i: \beta \rightarrow L_{a}^{2}$ is also weak*-weak* continuous. We omit the details.

(b) In [3], Anderson, Fernández, and Shields exhibit singular inner functions that are cyclic in $B\left(B_{0}\right)$. One has a complete description of the singular inner functions that are cyclic in $L_{a}^{2}$ from Korenblum [6] and Roberts [7] (see also Shapiro's notes [10]). Namely, the singular measure must put no mass on any closed set of $\partial D$ that is "thin" in the sense of Beurling, Carleson, and Hayman (see [4, p. 274] for these references and for the definition of the sets). It is still an open question as to whether this condition is sufficient for a singular inner function to be cyclic in $B$.

We conclude this paper with the following theorem.

THEOREM 3. If $f$ is an outer function in $\beta$, then $f$ is cyclic in $ß$.

Proof. Let

$$
g(z)=\exp \left\{\frac{1}{2 \pi} \int_{-\pi}^{\pi} \frac{e^{i t}+z}{e^{i t}-z} \log \left|g^{*}\left(e^{i t}\right)\right| d t\right\},
$$

where $f^{*}\left(e^{i t}\right)=f^{*}(t)=\lim _{r \uparrow 1} f\left(r e^{i t}\right)$ a.e. and

$$
\left|g^{*}\left(e^{i t}\right)\right|=\left|g^{*}(t)\right|= \begin{cases}1 & \text { if }\left|f^{*}(t)\right| \geq 1, \\ \left|f^{*}(t)\right| & \text { if }\left|f^{*}(t)\right| \leq 1 .\end{cases}
$$

We see that $g \in H^{\infty} \subset \mathbb{B}$ is an outer function and therefore cyclic in $\mathbb{B}$. Furthermore, 


$$
\begin{aligned}
|g(z)| & =\exp \left\{\frac{1}{2 \pi} \int_{-\pi}^{\pi} P_{z}(t) \log \left|g^{*}(t)\right| d t\right\} \\
& \leq \exp \left\{\frac{1}{2 \pi} \int_{-\pi}^{\pi} P_{z}(t) \log \left|f^{*}(t)\right| d t\right\}=|f(z)|, \quad z \in D,
\end{aligned}
$$

where $P_{z}$ is the Poisson kernel for the point $z$.

The result follows from Theorem 2 .

\section{References}

1. J. M. Anderson, Bloch functions: the basic theory, Operators and Function Theory (S. C. Power, ed.), pp. 1-17, Reidel, Dordrecht, 1985.

2. J. M. Anderson, J. Clunie, and Ch. Pommerenke, On Bloch functions and normal functions, J. Reine Angew. Math. 270 (1974), 12-37.

3. J. M. Anderson, J. L. Fernández, and A. L. Shields, Inner functions and cyclic vectors in the Bloch space, preprint.

4. L. Brown and A. L. Shields, Cyclic vectors in the Dirichlet space, Trans. Amer. Math. Soc. 285 (1984), 269-304.

5. P. L. Duren, B. W. Romberg, and A. L. Shields, Linear functionals on $H^{p}$ spaces with $0<p<1$, J. Reine Angew. Math. 238 (1969), 32-60.

6. B. I. Korenblum, Cyclic elements in some spaces of analytic functions, Bull. Amer. Math. Soc. (N.S.) 5 (1981), 317-318.

7. J. W. Roberts, Cyclic inner functions in the Bergman spaces and weak outer functions in $H^{p}, 0<p<1$, Illinois J. Math. 29 (1985), 25-38.

8. L. A. Rubel and A. L. Shields, The space of bounded analytic functions on a region, Ann. Inst. Fourier (Grenoble) 16 (1966), 235-277.

9. - The second duals of certain spaces of analytic functions, J. Austral. Math. Soc. Ser. A 11 (1970), 276-280.

10. J. Shapiro, Cyclic inner functions in Bergman spaces, preprint, 1980.

Leon Brown

Department of Mathematics

Wayne State University

Detroit, MI 48202
Allen L. Shields

Department of Mathematics

University of Michigan

Ann Arbor, MI 48109-1003 